Review Article

\title{
Food and Nutrition Security Potential of Anchote (Cocciniaabyssinica); A Tuberous Crop Indigenous to Ethiopia: A Review
}

\author{
Adugna Mosissa ${ }^{1}$, Yetenayet Bekele ${ }^{2}$, Tarekegn Berhanu ${ }^{3}$ \\ ${ }^{1}$ Department of Food Science and Nutrition, Wollega University, Shambu, Ethiopia \\ ${ }^{2}$ Department of Postharvest Management, Jimma University, Jimma, Ethiopia \\ ${ }^{3}$ Addis Ababa Science and Technology University, Addis Ababa, Ethiopia
}

Email address:

a.mosissa@yahoo.com (A. Mosissa)

\section{To cite this article:}

Adugna Mosissa, Yetenayet Bekele, Tarekegn Berhanu. Food and Nutrition Security Potential of Anchote (Cocciniaabyssinica); A Tuberous Crop Indigenous to Ethiopia: A Review. International Journal of Food Engineering and Technology. Vol. 7, No. 2, 2019 , pp. $34-38$. doi: $10.11648 /$ j.jfns.20190702.12

Received: March 5, 2019; Accepted: April 10, 2019; Published: June 28, 2019

\begin{abstract}
Root and tuber crops have potential contribution to the global food system in terms of food and nutrition security. They also contribute a major share in the traditional food systems of many people in Ethiopia. Anchote (Cocciniaabyssinica) is one of tuberous crops endemic in Ethiopia and originating from Southwestern parts of the country, particularly in East Wollega Zone of Oromia Region. The crop total yield is about 150-180 quintals per hectare. Nutritionally, anchote is a good source of carbohydrates, proteins, minerals and fibers. Its protein content is by far greater than other root crops and also contains adequate quality of essential amino acids. Despite its nutritional value and potential productivity, little attention is given to anchote to promote and develop as food crop. Furthermore, the crop has advantages in human nutrition, animal feed, medicinal, social/cultural, economic and industrial raw material; that makes it important food crop. Anchote is traditionally processed by boiling. When required, further processing (cooking) may also be applied prior to consumption. Except anchote stew (locally called "ittooancootee"), there is no value added commercial product produced from the crop. Therefore, the crop should be well adapted, promoted, researched and improved to support food security problem reduction and to alleviate malnutrition for the fast growing population of the country.
\end{abstract}

Keywords: Anchote (Cocciniaabyssinica), Food Security, Nutrition Security, Nutritional Composition

\section{Introduction}

Ethiopia has abundant natural food raw materials that can be used to alleviate malnutrition and food insecurity. Among these, root and tuber crops can be traced [1]. Though they are given little attention, the crops have potential contribution to the food system in terms of food security, nutrition and economy. The population of the country is growing fast and it is becoming difficult to assure food security and to alleviate malnutrition problems. For this reason it seems better to focus on indigenous crops with potential productivity, having good nutritional value and resisting harsh climatic conditions. Root and tuber crops have good resistance to difficult conditions such as soil acidity, soil infertility, and drought.

According to World Bank [2], climate and agricultural practices are among the causes of malnutrition in Ethiopia. In the country, there are some indigenous root and tuber crops [1, 3]. Among these, anchote (Cocciniaabyssinica) is highly productive and nutritionally ample crop. It is known to contain high calcium, carbohydrate and protein (with good amino acid composition) as well as sufficient other nutrients such as fiber, fat, and minerals compared to other commonly used root and tuber crops [4-6]. But its potential to support food and nutrition security is not exploited so far. The crop is among the underutilized but potentially productive and nutritious indigenous crops of Ethiopia. It is also a good yielding crop with short harvesting time $[4,7,8]$. 
However, anchote gets little research attention to promote its utilization and to extract sufficient information about its nature as a source of food for wider use in the country. It is only recently that attempts made to acknowledge advantages of the crop with regard to its agronomic performance and nutritional composition. The researchers also suggested the crop to be used as a food ingredient for new food product development and for different food formulations due to its potential nutrient content [6,9]. Consequently, this paper tried to present important available information about this valuable crop; and its potential uses as food source in reducing food insecurity and malnutrition.

\section{Methods}

To evaluate the potential of anchote in food and nutrition security, a narrative review was applied. Recent and specific papers about anchote were exhausted; especially, those related to its nutritional value. The historical background, agricultural and traditional development, current status and future perspectives of anchote as food crop have also been thoroughly assessed.

\section{Anchote as Food and Nutrition Security Crop}

Historical background, agricultural performance and traditional development, nutritional benefits, current development status and potential for nutritional contribution of anchote was compiled and presented here in brief.

\subsection{Nature and Agricultural Performance of Anchote}

The term "Anchote" is Afan Oromo name for Cocciniaabyssinica. It is an herbaceous, perennial, climbing plant whose stem is a vine like cucurbit particularly originating in East Wollega Zone of Oromia Region [3, 8]. Production of anchote still depends on local varieties maintained by farmers [1]. The crop is believed to have enormous genetic diversity, since it is indigenous and have been cultivated by native farmers for a long time [3]. Studies confirmed the existence of wide phenotypic variation in the species of anchote that provides good opportunity for genetic improvement of the crop through breeding [10]. Tolera [11] identified both common and rare morphological traits in different anchote accessions that indicate its broad genetic diversity and undergoes both sexual and asexual reproduction by seed and vegetative root, respectively. The same study also noted that flowers of separate sexes on the same plant with male flowers blooming earlier and this simplify cross-pollination. These double functions (sexual vs. asexual reproduction and separate male/female flowers) provide opportunities for breeding to improve the agronomic performance of the crop.

The cultivation of anchote is expanding to the southern parts of the country. Scholars evidenced that it is widely cultivated and used in East Wollega, West Wollega, Kelam
Wollega, Jimma, Illu Abba Bora, Kaffa, Welayita and Sidama zones [4, 8, 12]. The area covered by anchote in 1998/99 in East Wollega and West Wollega zones were reported as 440.75 ha and 440 ha respectively [13]. Also about 3000 ha of land was reported to be covered with anchote in West Wollega zone yielding about 25,000 tones product in 2011 [14]. The trend showed that production of the crop was increasing. Studies revealed that anchote is high yielding crop compared to the other root and tuber crops found in Ethiopia [14]. It is produced on several hectares of land in western Oromia zones, with an average yield varying from 100-180 quintals per hectare $[3,4,7]$.

Anchote can adapt to wide range of agro-ecological conditions [11]. And it can be grown with minimal inputs producing reasonable yields under conditions of low soil fertility, acidic soils, drought and intercropping with other crops $[8,12]$. The crop can be adapted to grow in an altitude range 1300 to $2800 \mathrm{~m}$. a. $\mathrm{s}$ with estimated annual precipitation range 762 to $1016 \mathrm{~mm}$ [14]. The available study reports recommended the crop to be promoted for greater consumption for human use to improve nutrition due to its good productivity [8]. Generally, the crop has promising yielding potential to be used as food security crop. Even the yield can be improved through scientific variety selection and breeding.

Anchote is cultivated for its tubers used to prepare different dishes in its native areas [15]. The farming, plantation, management, harvesting, storage and processing of the crop is traditional. Its tuber yield and composition was reported to be significantly affected by harvesting dates and in-situ storage [8]. The early harvest may result in low yield quantity and poor quality produce. The nutrient contents of the crop increase as it gets matured up to 7 months after planting [8]. Full maturity of anchote is attained in 3-5 months after planting [1, 8]. The time of maturity also depends on management practices and inputs [1]. The turning of its leaves to yellow is signaling for its maturity [8].

\subsection{Benefits of Anchote}

Root and tuber crops contribute a major share in the traditional food systems of many people in Ethiopia [16]. Specifically, anchote with its potential productivity has multidimensional advantages that encourage its development and utilization. Some of the diversified advantages of anchote are nutritional, economic, medicinal and social values $[12,17]$. Exceptionally, anchote has high nutritional value relative to other root and tuber crops $[5,8,9]$. For example; though protein is commonly nil in root and tuber crops, anchote contains relatively high protein with good amino acid composition [9]. In addition, the crop provides good composition of other important nutrients like $\mathrm{Ca}, \mathrm{K}$ and $\mathrm{Fe}$ beside the abundant carbohydrate and fiber content. It stores these nutrients in its edible parts, its tuber and leaf. For this reason researchers recommended that the crop should be promoted for greater consumption to improve nutrition [8-9]. Generally, anchote has a great nutritional advantage in order to support food and nutrition security in rural areas of the country. 
Regarding economic importance, anchote can be adapted and utilized by the rural small holder farmers with low cost and can supply important nutrients that may alleviate food and nutrition security. The most economical part of anchote is its diversified potentials in human nutrition, animal feed, medicinal applications and starch production, which will contribute towards food security, income generation, and resource base conservation $[9,18]$. The crop can be harvested in short period of time, and can stay in the soil when not immediately needed so that it can be used live for long time.

Anchote is traditionally used for medicinal purposes due to its natural composition. It was used to treat bone fracture, displaced joints and backache which is attributed to its high calcium content $[1,8,11]$. It is traditionally recommended to feed women that give birth due to the fact that it contains high $\mathrm{Ca}$ and $\mathrm{Fe}$ [8]. It can also be used to treat gonorrhea, tuberculosis and tumor cancer due to active ingredient contents $[11,17,19]$. Therefore, anchote is one of the food crops that help to maintain health of the consumers in addition to its nutritional value.

Anchote has also a cultural and social value among the producing areas. Mengeshaet al. [14] has reported that it is used as cultural and social crop for the farming community. Cultural dishes are prepared from mashed tubers by mixing with varies spices and butter [1]. This cultural food is consumed as one of diversified food staff especially during meskel (finding of the true cross) and New Year celebration of the country $[1,8]$. This shows that the producing society is using the crop as a culturally valuable food type.

\subsection{Nutritional Composition of Anchote}

Nutritionally, anchote is a good source of carbohydrates, proteins, minerals and fibers. Its protein content is by far greater than other root crops [9]. It is also rich source of calcium, which is an important constituent of our bones and teeth $[8,20]$. It was reported that the nutrient concentrations in anchote tuber are by far higher than that found in equal weight of potato (Solaniumtuberosum L.), yam (Dioscoreaabyssinica) and cassava (Manihotesculenta Crantz) tubers [5, 8]. Anchote could be a healthy food crop with potential source of equal or even better mineral elements as compared to other root and tuber crops [8]. Generally, the crop contains nutritionally valuable macro nutrients (carbohydrates, proteins, fiber, fats) and minerals such as $\mathrm{Ca}, \mathrm{K}, \mathrm{Na}, \mathrm{Fe}, \mathrm{Mg}, \mathrm{Zn}, \mathrm{Cu}, \mathrm{Co}, \mathrm{Mn}, \mathrm{S}, \mathrm{P}$, $\mathrm{N}$ and $\mathrm{Se}[4-5,8-9]$.

The proximate composition of anchote was studied in better detail than the other food parameters of the crop. There are physically identifiable types of anchote: white anchote and red anchote. Thered anchote is known for its higher protein and water content; and white anchote contains higher crude fat, crude fiber and total carbohydrate [5]. The proximate analysis of anchote indicated that it is a promising food source to solve the problem of malnutrition. As previous studies revealed, it contains both macro (those required in higher amount) and micro (those required in trace amount) nutrients. Parmar et al. [5] compared the mineral contents of anchote with cassava and sweet potato. Accordingly, anchote contains higher amounts of both macro and micro minerals than cassava and sweet potato, except $\mathrm{Na}$ and $\mathrm{K}$ [5]. Therefore, the crop can be used to fight against nutrition insecurity which is a challenging problem these days.

Anchote tuber contains higher protein relative to the commonly used tuberous crops potato, sweet potato and cassava $[9,18]$. The presence of crude protein may not suffice the quality of the protein. The amino acid composition analysis is important information to judge its quality. Ayalew [9] reported the amino acid composition of anchote tuber and compared with FAO/WHO standard for 1-2 years age children [9]. The study concluded that, anchote contains adequate quality of essential amino acids [6]. Therefore, the crop can be a source of good composition protein that can solve nutritional problems and it can also be used as additive to different food products. The nutritional quality of a protein food depends on the kinds and amounts of amino acids it contains, which represents a measure of the efficiency with which the body can utilize the protein. According to Ayalew et al. [6], anchote contains good quality protein composition and it can be used as a source of essential amino acids (Leu, Ile, Thr, SAAs, and AAAs) which are found in adequate amount to enhance protein quality especially when preparing plant-based complimentary food products. So, it is possible to conclude that anchote has reasonable quality of nutrients especially in composition. Therefore, it is a potential and promising source of nutrients that can be used in food formulations.

\subsection{Anti-Nutritional Factors in Anchote}

Anti-nutritional factors may reduce the nutrient utilization of plants or plant products used as foods. They can limit the digestibility and solubility of certain nutrients such as proteins, minerals and vitamins. Some anti-nutritional factors such as phytate, oxalate, tannin and cyanide in anchote were investigated $[4,7,9]$. Even raw anchote contains low levels of anti-nutrients except phytate, when compared to other roots and tubers such as potato, cocoyam, and taro [4, 7]. The cyanide content of anchote tuber falls far below the lethal dose of cyanide [9]. Fekadu [4] reported that the tannin content of raw anchote tuber is very low compared to its critical toxicity effect and further reduced during processing. Hence, its anti-nutritional effect may be insignificant in both raw and processed tuber. Despite this, since further processing of the food crop is required; the anti-nutrients could be reduced more to the safest level for consumption. Even traditionally anchote is not eaten raw where it is consumed.

\subsection{The Effect of Processing on Nutritional Value of Anchote}

In simple preparations of anchote, the tuber is lifted, washed, boiled, peeled, cut into small pieces and mixed with ground pepper and salt [1]. If more preparations needed, many spices and butter may be added. Then, the well prepared (spiced and buttered) food product may be eaten alone or with bread and/or "injera" [1]. This is the most frequently applied traditional method of anchote preparation. Previous studies 
reported that the crop is commonly processed traditionally by boiling after or before peeling [7]. When required, further processing (cooking) may also be applied prior to consumption. The traditional processing methods were found to be effective methods to reduce anti-nutrient contents in anchote tubers [4].

Shebabaw [20] reported the effect of three processing methods (boiling, roasting and fermentation) on anti-nutritional contents of anchote. According to this research, fermentation was the most efficient in reducing all the anti-nutritional factors (phytate, oxalate, tannin and cyanide) considered [20]. The boiling of anchote was stated to increase crude fiber content and improve the bioavailability of zinc [21]. On the other hand, processing was reported to decrease the crude protein, total ash, calcium, iron, and zinc content of anchote tubers [21]. Boiling before peeling of anchote tuber conserved majority of the nutrients since this method prevents them from leaching out into the cooking water. However, Crude fiber, Iron and Phosphorus were reported to be better preserved when boiling after peeling [4]. Fermentation of anchote tuber enhanced protein, fat, ash, Potassium $(K)$ and Zinc (Zn) content [20]. Only limited studies carried out on the effects of processing on the nutritional qualities of anchote.

\subsection{Limitations in Utilization of Anchote}

Integration of root and tuber crops in to the food system of the people may help to minimize the problem of food insecurity [14]. The full understanding of the economic, ecological and nutritional advantages of anchote is lacking in many parts of the producing region although anchote is familiar to the farmers in the regions [20]. MekbibandDeressa [1] listed the major broad spectrum factors limiting production and sustainable use of root and tuber crops like anchote. The factors mentioned are displacement by other crops, little research attention, drought, short shelf life, shortage of planting materials, limited knowledge of youth, and pests [1].

It was also noted that inappropriate agronomic packages, such as lack of recommended optimum fertilizer rates [3] and other agricultural practices may be some of the factors limiting productivity, quality and nutritive value of anchote. The study found that the lack of recommended optimum fertilizer rates and spacing overweigh the other agricultural practice problems in the case of anchote [3]. Generally, the main challenges for utilization of the crop in Ethiopia may be ignorance to the tuber crops, lack of awareness of the society and lack of support by policy direction.

\section{Conclusions}

Anchote (Cocciniaabyssinica) is a valuable food source with multidimensional qualities which makes it one of the most important crops to be further researched for enhanced utilization. It has a potential productivity and nutritional quality which could have advantages in feeding the fast growing population of the country. Overall, little has been studied about the crop and needs more research and development attention. If promoted in different areas, anchote could play a crucial role in improving the food and nutrition security of the society.

\section{Conflict of Interest}

The authors declared no conflict of interest.

\section{References}

[1] Mekbib, Y. \&Deressa, T. (2016). Exploration and collection of root and tuber crops in east Wollega and Ilu Ababora Zones: Rescuing declining genetic resource. Indian Journal of Traditional Knowledge, 15 (1), 86-92.

[2] The International Bank for Reconstruction and Development / The World Bank. (2012). Combating Malnutrition in Ethiopia, an Evidence-Based Approach for Sustained Results, Washington.

[3] Abera, G. \&Gudeta, H. (2007). Response of Anchote (CocciniaAbyssinica) to Organic and Inorganic Fertilizers Rates and Plant Population Density in Western Oromia, Ethiopia. East African Journal of Sciences, 1 (2), 120-126.

[4] Fekadu, H. (2014). Nutritional composition, antinutritional factors and effect of boiling on nutritional composition of Anchote (CocciniaAbyssinica) tubers. Food Science and Quality Management, 26, 25-38.

[5] Parmar, A., Gebre, A. B., Legesse, A., Demelash, Y., Fladung, K. and Hensel, O. (2017). Nutritional Comparison of White and Red CocciniaAbyssinica (Lam.) Cong. Accessions: An Under-Utilised Edible Tuber of the Ethiopian Highlands. Communication, Foods 2017, 6, 71.

[6] Ayalew, Y., Retta, N., Desse, G., Mohammed, A. \&Mellesse, A (2017). Amino acid profile and protein quality in tuber and leaf of Coccniaabyssinica (Lam.) (Cogn.) accessions of Ethiopia. Food SciNutr, 5, 722-729.

[7] Fekadu, H., Beyene, F. and Haki D. G. (2014). Evaluation of bioavailability and sensory preference of processed Anchote (CocciniaAbyssinica) tubers in Eastern Wollega, Ethiopia. Journal of Food and Nutrition Sciences, 2 (1), 1-12.

[8] Abera, G. \& Haile, D. (2015). Yield and nutrient concentration of Anchote [Cocciniaabyssinica (Lam.) Cogn.] affected by harvesting dates and in-situ storage. African Journal of Crop Science, 3 (5), 156-161.

[9] Ayalew, Y. (2016). Nutritional and Phytochemical Evaluation of Anchote (Cocciniaabyssinica) (Lam.) (Cogn.) Accessions to Promote its Contribution for Food Security and Medicinal Use. PhD Dissertation in Food Science and Nutrition, Addis Ababa University.

[10] Wondimu, T., Alamerew, S., Ayana, A. \&Garedew, W. (2014). Genetic Diversity Analysis among Anchote (Cocciniaabyssinica) Accessions in Western Ethiopia. International Journal of Agricultural Research, 9 (3), 149-157.

[11] Tolera, S. B. (2017). Morphological and Molecular Genetic Diversity and Cytogenetics of Cultivated Anchote (Cocciniaabyssinica (Lam.) Cogn) from Ethiopia. PhD Dissertation, Department of Microbial Cellular and Molecular Biology, Addis Ababa University. 
[12] Fekadu, D. (2011). Phenotypic and Nutritional Characterization of Anchote [Cocciniaabyssinica (Lam.) Cogn] Accessions of Ethiopia. M.Sc. Thesis, Jimma University, Ethiopia.

[13] Yassin, H., Mohammed, A., Fekadu, D., \&Hussen S. (2013). Effect of Flower Bud Removal on Growth and Yield of Anchote Root (Cocciniaabyssinica (Lam.) Cogn.) Accessions at Bishoftu. Advanced Research Journal of Plant and Animal Sciences, 1 (1), 7-13.

[14] Mengesha, D., Belew, D., Gebreselessie, W. and Sori, W. (2012). Growth and Yield Performance of Anchote [Cocciniaabyssinica (Lam.) Cogn.] in Response to Contrasting. Asian Journa of Plant Science, 11 (4), 172-181.

[15] Yambo Y. \&Feyissa T. (2013). Micropropagation of anchote [Cocciniaabyssinica (Lam.) Cogn.]: High calcium content tuber crop of Ethiopia. African Journal of Agricultural Research, 8 (46), 5915-5922.

[16] Edem, I. D \&Nkereuwem, M. E. (2015). Crucial Roles of Tuber Crops and the Development Activities in the Global Food System. American Journal of Agricultural Science, 2 (2), 42-49.

[17] Guma, B. T., Jane, K., Justus, O. \&Kariuki N. P. (2015).
Standardization Of In Vitro Sterilization and Callus Induction Protocol for Leaf Explants of Anchote: CocciniaAbyssinica. International Journal of Research and Development in Pharmacy and Life Sciences, 4 (2), 1427-1433.

[18] Desalegn, B. B., Tadesse, T. F., \& Haile, D. (2015). Physical and Proximate Characterization of Anchote (Cocciniaabyssinica) Accessions Grown under Hawassa and Wondo Genet Conditions, Southern Ethiopia. Food Science and Quality Management, 42, 62-74.

[19] Bekele, F., Abera, B. and Getahun, M. 2013. In vitro propagation of Anchote (Cocciniaabyssinica) (Lam.) Cogn.]. African Journal of Plant Science, 7 (6), 253-264.

[20] Shebabaw, H. (2013). Effect of Processing on Physicochemical and Antinutritional Factors of "Anchote"(CocciniaAbyssinica) and Development of Value Added Biscuit. Masters Thesis, Department of Chemical Engineering, Addis Ababa Institute of Technology.

[21] Fekadu, H., Beyene, F. \&Desse, G. (2013). Effect of Traditional Processing Methods on Nutritional Composition and Anti-nutritional Factors of Anchote (CocciniaAbyssinica (lam.) Cogn) Tubers Grown in Western Ethiopia. J Food Process Technol, 4 (7), 1-8. 